\title{
Transatlantica
}

Revue d'études américaines. American Studies Journal

\section{Les États-Unis à l'heure du choix. Entre conservatisme républicain et pragmatisme démocrate}

Université Grenoble-Alpes, 22 octobre 2020

\section{Meriem Naili}

\section{OpenEdition}

\section{Journals}

Édition électronique

URL : https://journals.openedition.org/transatlantica/15713

DOI : 10.4000/transatlantica. 15713

ISSN : 1765-2766

Éditeur

Association française d'Etudes Américaines (AFEA)

\section{Référence électronique}

Meriem Naili, « Les États-Unis à I'heure du choix. Entre conservatisme républicain et pragmatisme démocrate », Transatlantica [En ligne], 1 | 2020, mis en ligne le 01 décembre 2020, consulté le 01 février 2023. URL : http://journals.openedition.org/transatlantica/15713 ; DOI : https://doi.org/ 10.4000/transatlantica.15713

Ce document a été généré automatiquement le 1 février 2023.

\section{cc) (†) $९$}

Creative Commons - Attribution - Pas d'Utilisation Commerciale - Pas de Modification 4.0 International - CC BY-NC-ND 4.0

https://creativecommons.org/licenses/by-nc-nd/4.0/ 


\title{
Les États-Unis à l'heure du choix. Entre conservatisme républicain et pragmatisme démocrate
}

Université Grenoble-Alpes, 22 octobre 2020

\author{
Meriem Naili
}

\section{Introduction}

1 La journée d'études qui a eu lieu le jeudi 22 octobre à l'université Grenoble Alpes a tenté de mettre en avant la particularité des élections américaines du 3 novembre 2020, douze jours avant leur tenue. Les intervenants ont souligné l'affaiblissement du fonctionnement démocratique du pays par un président qui a usé de son pouvoir au point d'être remis en cause devant les plus hautes juridictions lors d'un procès en destitution. Dans un contexte de crise sanitaire sans précédent, cette élection verra le plus vieux président jamais porté à cette fonction, quelle que soit l'issue du scrutin. Audelà des enjeux politiques, économiques, environnementaux et sociétaux que cette élection met en exergue, les participants à cette journée d'études ont su tracer les contours d'une crise identitaire profonde, dont les véritables répercussions ne pourront se mesurer qu'après le 3 novembre.

2 La journée a vu se succéder divers spécialistes de l'Amérique du Nord, réunis en trois panels thématiques. Les interventions ont, dans un premier temps, identifié et défini le concept nouveau de trumpisme, placé dans un contexte historique particulier, et mesuré son impact sur le futur de la démocratie libérale américaine (panel 1). Les intervenants se sont également penchés sur les conséquences profondes de ce nouveau concept, à la fois sur l'identité et sur la stratégie démocrates pour cette élection historique (panel 3). Le Minnesota aura enfin servi de cas d'étude afin d'illustrer les différentes problématiques que ce nouveau déséquilibre idéologique, créé par la "présidence impériale " de Donald Trump, a soulevées (panel 2). Enfin les interventions de deux keynotes, Francois Vergniolle de Chantal (Université de Paris) et Elisabeth Vallet 
(Université du Québec à Montréal), ont placé la discussion dans un contexte historique et géographique plus large.

\section{Le trumpisme : bilan et perspective d'une présidence pas comme les autres}

3 Dans sa conférence plénière d'ouverture, Francois Vergniolle de Chantal rappelle les cadres politique et sociétal, les rapports de force dans lesquels se joue cette élection, ainsi que la littérature de science politique entourant le concept de trumpisme. Il souligne que la présidence de Donald Trump «a brouillé l'identité du parti républicain", mais que, malgré l'absence d'un rassemblement de cœur et d'une trumpisation idéologique au début de son mandat, un alignement instrumental s'est opéré avec les élus républicains. La présentation souligne bien le positionnement opportuniste de Donald Trump: son horizon d'action et son caractère populiste s'expriment en amont de toute idéologie et portent à croire que le mouvement trumpiste n'est que transitoire. Cependant cette vacuité doctrinale de la présidence Trump est toxique pour le parti et Francois Vergniolle de Chantal identifie clairement les enjeux de l'élection à venir au-delà du combat de personnes et de personnalités, pour une redéfinition partisane du républicanisme.

4 Au travers d'un rappel historique visant à contextualiser historiquement le trumpisme, le premier paneliste, Olivier Burtin (Université Louis-et-Maximilien de Munich) interroge pour sa part la notion de "présidence impériale ». Il souligne que Donald Trump n'est pas le premier président à mentir, à venir du monde médiatique (Reagan) ou à essayer d'utiliser les questions migratoires comme outil électoral. Sa présidence met en lumière les faiblesses, plus que les forces, de la fonction présidentielle: difficulté d'arriver à des accords bipartisans, attitude de plus en plus critique des médias ou diminution du prestige des États-Unis sur la scène internationale. ${ }^{1}$ Enfin, il rappelle justement le procès en destitution et son rôle catalyseur dans la modération du pouvoir exécutif par les institutions, notamment en cas de victoire de Joe Biden.

Enfin, les deux autres intervenants de ce panel apportent une analyse plus approfondie des enjeux aux niveaux local (Douglas, Arizona) et international. Cléa Fortuné (Université Paris 3 - Sorbonne Nouvelle) livre un très bon aperçu des caractéristiques de l'Arizona qui en font un swing state. En effet, Donald Trump y a récolté $48 \%$ des voix populaires en 2016, quand Joe Biden recueillerait $49 \%$ d'intentions de vote en 2020. La démographie de l'Arizona évolue rapidement et l'exode des jeunes populations vers les villes comme Phoenix, ainsi qu'une croissance de la population hispanique (surpassant en nombre les électeurs africains-américains pour la première fois au niveau national) constituent des éléments clés de l'équation électorale en Arizona. Elle rappelle d'ailleurs qu'il s'agirait d'une première victoire pour les Démocrates dans cet État en 24 ans mais que, malgré des propositions radicalement différentes des deux grands partis, l'issue n'aura pas forcément d'impact significatif sur la sécurité frontalière. Plus globalement, Jean-Marie Ruiz (Université Savoie Mont Blanc) rappelle que les enjeux de cette élection se prolongent bien au-delà de la simple confrontation de programmes. Il souligne que c'est la nature de la démocratie américaine qui est en jeu. Sur le plan intérieur, une victoire de Donald Trump renforcerait les aspects «illibéraux » de la fonction présidentielle: personnalisation du pouvoir, opposition décomplexée aux contre-pouvoirs (médias, parti d'opposition, politisation des cours fédérales, etc.) et 
populisme. Sur le plan extérieur, elle exacerberait la fin d'un ordre internationaliste libéral : déclin du multilatéralisme et du libre-échange, politique étrangère indifférente à la démocratie ou encore affinités avec les régimes autoritaires.

6 Toutes ces analyses ne sont pas sans rappeler l'importance qu'une victoire ou une défaite du président sortant aurait sur la signification même du courant politicoidéologique qu'il a tenté d'affirmer au cours des quatre dernières années. En face, le camp démocrate a dû tirer les leçons nécessaires afin de mettre un terme à ce mouvement si souvent décrit comme éphémère. Les analyses présentées lors du troisième panel n'ont pas manqué de rappeler une polarisation alarmante d'une société et d'un corps électoral en proie à une crise identitaire majeure.

\section{Une opposition démocrate contrainte à une restructuration plus inclusive?}

7 Dans une analyse plutôt historique du mode de désignation du candidat représentant chaque parti, Raphaël Ricaud (Université Paul-Valery) s'interroge, à juste titre, sur le caractère démocratique du système des caucus encore employé par le parti démocrate dans certains États comme l'Iowa. Désormais remplacé par un système de primaires dans la plupart des États fédérés, ce mode de sélection du candidat représentant le parti au niveau national a été critiqué notamment pour sa tendance à favoriser le clientélisme. Historiquement, l'Iowa a tout de même souvent joué un rôle central et servi de rampe de lancement pour le candidat démocrate désigné. Loin d'avoir été révélateurs, les résultats de ce caucus soulignent les particularités de cette année électorale. Arrivé quatrième, Joe Biden n'aura en effet pas atteint le seuil de viabilité (15\%) dans certains comtés. L'Iowa n'a donc pas joué son rôle d'indicateur et ses habitants ont préféré mettre en avant de nouveaux candidats. La question qui se pose alors en cas d'élection de Joe Biden à la Maison-Blanche est de savoir s'il sera un passeur de flambeau au sein d'un parti démocrate en pleine évolution, ou le dernier représentant d'une vieille génération de politiciens en voie de disparition. À ce propos, Francois Vergniolle de Chantal a estimé dans son introduction que Kamala Harris (censée représenter l'Amérique du futur) ne tire pas suffisamment profit de son statut de candidate issue des minorités et ne porte pas assez fort le flambeau qui lui a été tendu, dans une démarche constituant un risque potentiel pour les Démocrates.

Thomas Jeangirard (Université d'Orléans), pour sa part, produit une description pertinente des dynamiques de fond qui ont animé le parti démocrate ces dernières années et porté ce dernier plus « à gauche ». En prenant l'exemple de Bernie Sanders, il décrit la résurgence d'un courant socialiste au sein du parti, grâce à des mouvements mobilisateurs - notamment anti-mondialisation - tels que Occupy Wall Street. Il rappelle cependant que dans le contexte américain, le pragmatisme électoral prime sur les principes idéologiques. Les deux grands partis ont, en effet, imposé des règles très strictes pour éviter que les petits partis soient sur les ballots. Ainsi, des candidats alternatifs soutenus par certaines organisations telles que les Democratic Socialists of America ou le Working Family Party ont du mal à déstabiliser l'establishment du parti démocrate. D'autres raisons expliquent également cette "dictature du bipartisme», telles que la décentralisation organisationnelle des partis (avec une ligne partisane dirigée par les financements) accompagnée d'un opportunisme politique (en fonction de ce que les électeurs veulent), ou encore l'absence de second tour. Tous ces éléments 
poussent, de fait, les électeurs à opter pour un vote stratégique face à deux uniques options : la confrontation ou le réalignement. La première encouragerait une rupture avec le programme du parti démocrate et un rapprochement avec de plus petits partis (Greens ou New People's Party) en utilisant les primaires comme plateforme de discussion pour des sujets chers à la doctrine socialiste, et attirer ainsi de nouveaux électeurs. La seconde préconise de symétriser cette polarisation politique au sein du parti en redéfinissant un nouveau projet commun. Le choix de Joe Biden pour représenter le parti et l'issue du scrutin final sont, en ce sens, autant d'éléments qui vont dans le sens de ce réalignement.

\section{Le Minnesota, un État au centre des attentions}

9 L'intérêt porté sur l'État du Minnesota s'explique, à juste titre, par le décès de George Floyd le 25 mai 2020 à Minneapolis. Cependant, comme le remarquent Martine Azuelos et Cynthia Ghorra Gobin (Université Paris 3), l'intérêt de Donald Trump pour cet État est antérieur à cet évènement. En effet, dans un État qui ne compte pourtant que dix Grands Électeurs, la différence de voix n'était que très infime en 2016, 45000 voix seulement séparant Trump de sa concurrente. Cet écart n'a fait que diminuer depuis 2008, ce qui a conduit Donald Trump à penser qu'il pouvait l'emporter en augmentant sa présence et sa visibilité dans un État divisé en deux et où «les villes jumelles (Minneapolis-Saint Paul) votent de façon aussi progressiste que la Californie, alors que certaines circonscriptions votent de manière aussi conservatrice que l'Alabama ». Les évolutions politiques reposent, en effet, sur les évolutions économiques et démographiques de l'État. Les activités de services représentent $80 \%$ du PIB du Minnesota, l'agriculture seulement $2 \%$ et les services manufacturiers $18 \%$. Les résultats seront donc révélateurs de la stratégie de Donald Trump selon Martine Azuelos, et témoigneront - ou non - d'une volonté des électeurs de renforcer la présence républicaine dans cet État.

De son coté, Loïc Laroche (Institut Catholique de Lille) rappelle que dans une Amérique divisée et polarisée, l'information est un enjeu majeur dans les élections. Les médias traditionnels se veulent neutres et ne se positionnent généralement pas contre l'un ou l'autre des candidats. Paradoxalement, ils sont attaqués par les deux camps pour leur absence d'engagement dans certains cas extrêmes comme les manifestations et émeutes qui ont suivies la mort de George Floyd. Loïc Laroche énumère les évènements et/ou affaires qui ont alimenté les guerres culturelles récentes dans la presse américaine et qui s'invitent dans la campagne présidentielle, notamment l'affaire Tom Cotton du New York Times. Il décrit ainsi une évolution des normes du discours journalistique entre les trois sphères classiques du consensus, de la déviance et de la controverse légitime - évolution au cours de laquelle le mouvement Black Lives Matter aura ainsi fait finalement irruption dans la sphère du consensus. Ces changements de normes du discours journalistique posent le risque d'une triple cassure selon lui: générationnelle, sociale et idéologique. Le péril, avec une réélection de Donal Trump, est l'aggravation de l'intolérance et une plus grande polarisation risquant d'accentuer encore davantage ces cassures. Le cas de George Floyd et les enjeux identitaires qui entourent les élections de cet automne dans le Minnesota, et plus largement dans tout le pays, rappellent l'importance du rôle des médias comme quatrième pouvoir dans une société de plus en plus polarisée et une démocratie fragilisée. 


\section{Conclusion}

11 La journée d'études du 22 octobre qui s'est tenue à l'UGA a su mettre en avant les failles du mode de scrutin américain, ainsi que l'irréconciliabilité des deux principaux groupes de votants appelés à y participer. Une défaite de Donald Trump n'est pas forcément synonyme d'une défaite du trumpisme, et une victoire de Joe Biden ne marque pas forcément le renouvellement du parti démocrate sur une base plus socialiste. La culture du compromis a été très ébranlée par les manifestations qui ont suivi la mort de George Floyd et durant lesquelles les vieux clivages idéologiques, voire identitaires, ont refait surface. La conclusion des débats par Elisabeth Vallet a su anticiper les conséquences d'un scrutin sous tension (en particulier, un dépouillement mouvementé et vite instrumentalisé par Trump), dans le contexte d'une pandémie hors de contrôle. Cependant le parallèle fait avec le Canada aurait pu être élargi a bien d'autres États, notamment européens, alliés historiques des États-Unis qui ont également souffert de l'unilatéralisme maladif de la politique étrangère trumpiste. Raison pour laquelle leurs yeux sont intensément rivés vers l'autre rive de l'Atlantique.

\section{NOTES}

1. Notamment à la suite des retraits successifs du traité de Paris sur le climat, de l'Organisation Mondiale de la santé ou des accords avec l'Iran.

\section{RÉSUMÉS}

Entre le bilan d'une présidence républicaine marquée par l'apparition et l'affirmation du "trumpisme» et une opposition démocrate qui a dû se relancer structurellement et idéologiquement au lendemain de l'élection de Donald Trump en 2016, les enjeux de l'élection du 3 novembre 2020 vont au-delà d'une simple confrontation partisane. Comme les évènements de Minneapolis du 25 mai 2020 et le décès de George Floyd l'ont révélé, l'Amérique est en proie à une crise identitaire profonde. Quelles sont les options qui se font face et, plus important encore, quels sont les réels enjeux de ce scrutin historique ? À l'heure du choix, cette journée d'études nous offre une présentation dynamique des différentes perspectives et problématiques auxquelles fait face l'Amérique d'aujourd'hui.

Far from a mere partisan contest, the election of November 3, 2020 represented a moment of truth for a Republican presidency marked by the appearance and affirmation of "Trumpism" and a Democratic opposition on the path to structural and ideological reinvention ever since Donald 
Trump's 2016 election. As the events of Minneapolis of May 25, 2020 and the passing of George Floyd revealed, America is in the thralls of a deep identity crisis. What are the options open to voters and, more importantly, what are the real issues that lie behind this historic election? At this historic juncture, this seminar gave us a dynamic presentation of the different perspectives and issues facing America today.

INDEX

Keywords : Trumpism, Democrats, presidential elections, democracy, identity crisis Mots-clés : trumpisme, Démocrates, élections présidentielles, démocratie, crise identitaire Thèmes : Actualité de la recherche

\section{AUTEUR}

\section{MERIEM NAILI}

University of Exeter, Department of Politics 